\title{
Correction to: A novel impedimetric sensor for trace level detection of dimethyl sulfide (DMS)
}

\author{
Hassan Iden ${ }^{1,2} \cdot$ Ricardo Adriano Dorledo de Faria $^{1,3}$ (1) $\cdot$ Luiz Guilherme Dias Heneine ${ }^{4}$ Tulio Matencio ${ }^{5}$. \\ Younès Messaddeq ${ }^{1,6}$
}

Published online: 20 August 2020

○) Springer Science+Business Media, LLC, part of Springer Nature 2020

\section{Correction to: \\ Journal of Materials Science: Materials in Electronics (2020) \\ https://doi.org/10.1007/s10854-020-03588-0}

The original version of this paper was unfortunately published with errors which are corrected by publishing this Correction article.

Errors have subsequently been identified in the original publication, and the following corrections should be noted in Eqs. 3, 4, 5, 6, and 7. These are corrected below.

Equation 3 should be

$\mathrm{LOD}=2\left(\frac{s_{y} t}{a_{1}}\right) \sqrt{\left(\frac{1}{N}\right)+1+\frac{\left(y_{c}-\bar{y}\right)^{2}}{a_{1}^{2} \sum_{i=1}^{n}\left(x_{i}-\bar{x}\right)^{2}}}$.

Equation 4 should be

The original article can be found online at https://doi.org/10.1007/ s10854-020-03588-0.

Younès Messaddeq

younes.messaddeq@copl.ulaval.c

1 Center for Optics, Photonics and Lasers (COPL), Université Laval, Quebec, Canada

2 CDN Isotopes, Montreal, Canada

3 Department of Chemical Engineering, Universidade Federal de Minas Gerais (UFMG), Minas Gerais, Brazil

4 Laboratory of Applied Immunology, Fundação Ezequiel Dias (FUNED), Minas Gerais, Brazil

5 Department of Chemistry, Universidade Federal de Minas Gerais (UFMG), Minas Gerais, Brazil

6 Institute of Chemistry, UNESP, São Paulo, Brazil

$$
y_{c}=a_{0}+s_{y} t \sqrt{\left(\frac{1}{N}\right)+1+\frac{\bar{x}^{2}}{\sum_{i=1}^{n}\left(x_{i}-\bar{x}\right)^{2}}} .
$$

Equation 5 should be

$$
\mathrm{LOQ}=\left(\frac{y-a_{0}}{a_{1}}\right)+\left(\frac{s_{y} t}{a_{1}}\right) \sqrt{\left(\frac{1}{N}\right)+1+\frac{\left(y_{h}-\bar{y}\right)^{2}}{a_{1}^{2} \sum_{i=1}^{n}\left(x_{i}-\bar{x}\right)^{2}}} .
$$

Equation 6 should be

$$
x_{c}=\left(\frac{s_{y} t}{a_{1}}\right) \sqrt{\left(\frac{1}{N}\right)+1+\frac{\bar{x}^{2}}{\sum_{i=1}^{n}\left(x_{i}-\bar{x}\right)^{2}}} .
$$

Equation 7 should be

$$
y_{h}=a_{0}+2 s_{y} t \sqrt{\left(\frac{1}{N}\right)+1+\frac{\left(x_{c}-\bar{x}\right)^{2}}{\sum_{i=1}^{n}\left(x_{i}-\bar{x}\right)^{2}}} .
$$

Publisher's Note Springer Nature remains neutral with regard to jurisdictional claims in published maps and institutional affiliations. 